\title{
Stability and Convergence of an Effective Finite Element Method for Multiterm Fractional Partial Differential Equations
}

\author{
Jingjun Zhao, Jingyu Xiao, and Yang Xu \\ Department of Mathematics, Harbin Institute of Technology, Harbin 150001, China \\ Correspondence should be addressed to Yang Xu; yangx@hit.edu.cn \\ Received 11 December 2012; Revised 3 February 2013; Accepted 6 February 2013 \\ Academic Editor: Dragoş-Pătru Covei
}

Copyright (C) 2013 Jingjun Zhao et al. This is an open access article distributed under the Creative Commons Attribution License, which permits unrestricted use, distribution, and reproduction in any medium, provided the original work is properly cited.

\begin{abstract}
A finite element method (FEM) for multiterm fractional partial differential equations (MT-FPDEs) is studied for obtaining a numerical solution effectively. The weak formulation for MT-FPDEs and the existence and uniqueness of the weak solutions are obtained by the well-known Lax-Milgram theorem. The Diethelm fractional backward difference method (DFBDM), based on quadrature for the time discretization, and FEM for the spatial discretization have been applied to MT-FPDEs. The stability and convergence for numerical methods are discussed. The numerical examples are given to match well with the main conclusions.
\end{abstract}

\section{Introduction}

In recent years, the numerical treatment and supporting analysis of fractional order differential equations has become an important research topic that offers great potential. The FEMs for fractional partial differential equations have been studied by many authors (see [1-3]). All of these papers only considered single-term fractional equations, where they only had one fractional differential operator. In this paper, we consider the MT-FPDEs, which include more than one fractional derivative. Some authors also considered solving linear problems with multiterm fractional derivatives (see $[4,5])$. This motivates us to consider their effective numerical solutions for such MT-FPDEs, which have been proposed in $[6,7]$.

Let $\Omega=(0, X)^{d}$, where $d \geq 1$ is the space dimension. We consider the MT-FPDEs with the Caputo time fractional derivatives as follows:

$$
P\left({ }^{C} D_{t}\right) u(t, x)-\Delta_{x} u(t, x)=f(t, x), \quad t \in[0, T], x \in \Omega,
$$

$$
\begin{gathered}
u(0, x)=u_{0}(x), \quad x \in \Omega, \\
u(t, x)=0, \quad t \in[0, T], x \in \partial \Omega,
\end{gathered}
$$

where the operator $P\left({ }^{C} D_{t}\right) u(t, x)$ is defined as

$$
P\left({ }^{C} D_{t}\right) u(t, x)=\left({ }_{0}^{C} D_{t}^{\alpha}+\sum_{i=1}^{s} a_{i 0}^{C} D_{t}^{\alpha_{i}}\right) u(t, x)
$$

with $0<\alpha_{s}<\alpha_{s-1}<\cdots<\alpha_{1}<\alpha<1$ and $\left\{a_{i}>0\right\}_{i=1}^{s}$. Here ${ }_{0}^{C} D_{t}^{\alpha} u(t, x)$ denotes the left Caputo fractional derivative with respect to the time variable $t$ and $\Delta_{x}$ denotes the Laplace operator with respect to the space variable $x$.

Some numerical methods have been considered for solving the multiterm fractional differential equations. In [8], Liu et al. investigate some effective numerical methods for time fractional wave-diffusion and diffusion equations:

$$
{ }_{0}^{C} D_{t}^{\alpha} u(t, x)-k \Delta_{x} u(t, x)=f(t, x), \quad 0<x<L, t>0,
$$

where $k$ and $L$ are arbitrary positive constants and $f(t, x)$ is a sufficiently smooth function. The authors consider the implicit finite difference methods (FDMs) and prove that it is unconditionally stable. The error estimate of the FDM is $O\left(\Delta t+\Delta t^{2-\alpha}+\Delta x\right)$, where $\Delta t$ and $\Delta x$ are the time and space step size, respectively. They also investigate the fractional predictor-corrector methods (FPCMs) of the AdamsMoulton methods for multiterm time fractional differential equations (1) with order $\left\{\alpha_{i}\right\}_{i=1, \ldots .,}$ by solving the equivalent 
Volterra integral equations. The error estimate of the FPCM is $O\left(\Delta t+\Delta t^{1+\min \left\{\alpha_{l}\right\}}+\Delta x^{2}\right)$. In recent years, there are some articles for the predictor-correction method for initial-value problems (see [9-14]). For the application of the FDMs, there have been many research articles as follows. In [15-20], Simos et al. investigate the numerical methods for solving the Schrödinger equation. In [21-24], the Runge-Kutta methods are considered and applied to get the numerical solution of orbital problems. For long-time integration, the NewtonCotes formulae are considered in [25-27].

In [28], Badr investigate the FEM for linear multiterm fractional differential equations with one variable as follows:

$$
\begin{array}{r}
{ }_{0}^{C} D_{t}^{1+\alpha} u(t)+\sum_{i=1}^{s} A_{i}(x) D^{\alpha_{i}} u(x)=f(t), \quad \alpha \leq n, \\
\alpha_{i}<n-1, \quad 0<t<1,
\end{array}
$$

where $A_{i}(x)$ are known functions. The author gives the details of the modified Galerkin method for the above equations and makes the numerical example for checking the numerical method. In [29], Ford et al. consider the FEM for (5) with singular fractional order and obtain the error estimate $O\left(\Delta t^{2-\alpha}+\Delta x^{2}\right)$. In this paper, we follow the work in [29] and consider the FEM for solving MT-FPDEs (1)-(3). Then, we prove the stability and convergence of the FEM for MTFPDEs and make the error estimate.

The paper is organized as follows. In Section 2, the weak formulation of the MT-FPDEs is given and the existence and uniqueness results for such problems are proved. In Section 3, we consider the convergence rate of time discretization of MT-FPDEs, based on the Diethelm fractional backward difference method (DFBDM). In Section 4, we propose an FEM based on the weak formulation and carry out the error analysis. In Section 5, the stability of this method is proven. Finally, the numerical examples are considered for matching well with the main conclusions.

\section{Existence and Uniqueness}

Let $\Gamma(\cdot)$ denote the gamma function. For any positive integer $n$ and $n-1<\alpha<n$, the Caputo derivative are the RiemannLiouville derivative are, respectively, defined as follows [30].

(i) The left Caputo derivatives:

$$
{ }_{0}^{C} D_{t}^{\alpha} v(t):=\frac{1}{\Gamma(n-\alpha)} \int_{0}^{t} \frac{1}{(t-\tau)^{\alpha-n+1}}\left(\frac{d^{n}}{d \tau^{n}} v(\tau)\right) d \tau .
$$

(ii) The left Riemann-Liouville derivatives:

$$
{ }_{0}^{R} D_{t}^{\alpha} v(t):=\frac{1}{\Gamma(n-\alpha)} \frac{d^{n}}{d t^{n}} \int_{0}^{t} \frac{v(\tau)}{(t-\tau)^{\alpha-n+1}} d \tau
$$

(iii) The right Riemann-Liouville derivatives:

$$
{ }_{t}^{R} D_{T}^{\alpha} v(t):=\frac{(-1)^{n}}{\Gamma(n-\alpha)} \frac{d^{n}}{d t^{n}} \int_{t}^{T} \frac{v(\tau)}{(\tau-t)^{\alpha-n+1}} d \tau .
$$

Let $C^{\infty}(0, T)$ denote the space of infinitely differentiable functions on $(0, T)$ and $C_{0}^{\infty}(0, T)$ denote the space of infinitely differentiable functions with compact support in $(0, T)$. We use the expression $A \leqslant B$ to mean that $A \leq c B$ when $c$ is a positive real number and use the expression $A \cong B$ to mean that $A \lesssim B \lesssim A$. Let $L_{2}(\mathbb{Q})$ be the space of measurable functions whose square is the Lebesgue integrable in $Q$ which may denote a domain $Q=I \times \Omega, I$ or $\Omega$. Here time domain $I:=(0, T)$ and space domain $\Omega:=(0, X)$. The inner product and norm of $L_{2}(Q)$ are defined by

$$
\begin{array}{r}
(u, v)_{L_{2}(\mathbb{Q})}:=\int_{\mathscr{Q}} u v d Q, \quad\|u\|_{L_{2}(Q)}:=(u, u)_{L_{2}(\mathbb{Q})}^{1 / 2}, \\
\forall u, v \in L_{2}(\mathcal{Q}) .
\end{array}
$$

For any real $\sigma>0$, we define the spaces ${ }^{l} H_{0}^{\sigma}(Q)$ and ${ }^{r} H_{0}^{\sigma}(Q)$ to be the closure of $C_{0}^{\infty}(Q)$ with respect to the norms $\|v\|_{{ }^{l} H_{0}^{\sigma}(\mathscr{Q})}$, and $\|v\|_{{ }^{r} H_{0}^{\sigma}(Q)}$ respectively, where

$$
\begin{aligned}
& \|v\|_{{ } H_{0}^{\sigma}(Q)}:=\left(\|v\|_{L_{2}(Q)}^{2}+|v|^{2}{ }_{H_{0}^{\sigma}(\mathcal{Q})}\right)^{1 / 2}, \\
& |v|_{{ } H_{0}^{\sigma}(Q)}^{2}:=\left\|{ }_{0}^{R} D_{t}^{\sigma} v\right\|_{L_{2}(Q)}^{2}, \\
& \|v\|{ }_{{ }^{r} H_{0}^{\sigma}(Q)}:=\left(\|v\|_{L_{2}(Q)}^{2}+|v|_{{ }^{r} H_{0}^{\sigma}(Q)}^{2}\right)^{1 / 2}, \\
& |v|_{{ }^{r} H_{0}^{\sigma}(Q)}^{2}:=\left\|{ }_{t}^{R} D_{T}^{\sigma} v\right\|_{L_{2}(Q)}^{2} .
\end{aligned}
$$

In the usual Sobolev space $H_{0}^{\sigma}(Q)$, we also have the definition

$$
\begin{aligned}
\|v\|_{H_{0}^{\sigma}(Q)} & :=\left(\|v\|_{L_{2}(Q)}^{2}+|v|_{H_{0}^{\sigma}(Q)}^{2}\right)^{1 / 2}, \\
|v|_{H_{0}^{\sigma}(Q)}^{2} & :=\frac{\left({ }_{0}^{R} D_{t}^{\sigma} v,{ }_{t}^{R} D_{T}^{\sigma} v\right)_{L_{2}(Q)}}{\cos (\pi \sigma)} .
\end{aligned}
$$

From [3], for $\sigma>0, \sigma \neq n-1 / 2$, the spaces ${ }^{l} H_{0}^{\sigma}(\mathbb{Q})$, ${ }^{r} H_{0}^{\sigma}(Q)$, and $H_{0}^{\sigma}(\mathcal{Q})$ are equal, and their seminorms are all equivalent to $|\cdot|_{H_{0}^{\sigma}(Q)}$. We first recall the following results.

Lemma 1 (see [3]). Let $0<\theta<2, \theta \neq 1$. Then for any $w, v \in$ $H_{0}^{\theta / 2}(0, T)$, then

$$
\left({ }_{0}^{R} D_{t}^{\theta} w, v\right)_{L_{2}(0, T)}=\left({ }_{0}^{R} D_{t}^{\theta / 2} w,{ }_{t}^{R} D_{T}^{\theta / 2} v\right)_{L_{2}(0, T)} .
$$

From [3], we define the following space:

$$
\begin{aligned}
B^{\alpha / 2}(I \times \Omega)= & H^{\alpha / 2}\left(I, L_{2}(\Omega)\right) \cap H^{\alpha_{1} / 2}\left(I, L_{2}(\Omega)\right) \cap \cdots \\
& \cap H^{\alpha_{s} / 2}\left(I, L_{2}(\Omega)\right) \cap L_{2}\left(I, H_{0}^{1}(\Omega)\right) \\
= & H^{\alpha / 2}\left(I, L_{2}(\Omega)\right) \cap L_{2}\left(I, H_{0}^{1}(\Omega)\right) .
\end{aligned}
$$


Here $B^{\alpha / 2}(I \times \Omega)$ is a Banach space with respect to the following norm:

$$
\|v\|_{B^{\alpha / 2}(I \times \Omega)}=\left(\|v\|_{H^{\alpha / 2}\left(I, L_{2}(\Omega)\right)}^{2}+\|v\|_{L_{2}\left(I, H_{0}^{1}(\Omega)\right)}^{2}\right)^{1 / 2},
$$

where $H^{\alpha / 2}\left(I, L_{2}(\Omega)\right):=\left\{v ;\|v(t, \cdot)\|_{L_{2}(\Omega)} \in H^{\alpha / 2}(I)\right\}$, endowed with the norm

$$
\|v\|_{H^{\alpha / 2}\left(I, L_{2}(\Omega)\right)}:=\|\| v(t, \cdot)\left\|_{L_{2}(\Omega)}\right\|_{H^{\alpha / 2}(I)} .
$$

Based on the relation equation between the left Caputo and the Riemann-Liouville derivative in [31], we can translate the Caputo problem to the Riemann-Liouville problem. Then, we consider the weak formulation of (1) as follows. For $f \in B^{\alpha / 2}(I \times \Omega)^{\prime}$, find $u(t, x) \in B^{\alpha / 2}(I \times \Omega)$ such that

$$
\mathscr{A}(u, v)=\mathscr{F}(v), \quad v \in B^{\alpha / 2}(I \times \Omega),
$$

where the bilinear form is, by Lemma 1 ,

$$
\begin{aligned}
\mathscr{A}(u, v):= & \left({ }_{0}^{R} D_{t}^{\alpha / 2} u,{ }_{t}^{R} D_{T}^{\alpha / 2} v\right)_{L_{2}(I \times \Omega)} \\
& +\sum_{i=1}^{s} a_{i}\left({ }_{0}^{R} D_{t}^{\alpha_{i} / 2} u,{ }_{t}^{R} D_{T}^{\alpha_{i} / 2} v\right)_{L_{2}(I \times \Omega)} \\
& +\left(\nabla_{x} u, \nabla_{x} v\right)_{L_{2}(I \times \Omega)},
\end{aligned}
$$

and the functional is $\mathscr{F}(v):=(\bar{f}, v)_{L_{2}(I \times \Omega)}, \bar{f}(t, x):=f(t, x)+$ $\left(u_{0}(x) t^{-\alpha} / \Gamma(1-\alpha)\right)+\sum_{i=1}^{s} a_{i}\left(u_{0}(x) t^{-\alpha_{i}} / \Gamma\left(1-\alpha_{i}\right)\right)$.

Based on the main results in Subsection 3.2 in [32], we can prove the following existence and uniqueness theorem.

Theorem 2. Assume that $0<\alpha<1$ and $\bar{f} \in B^{\alpha / 2}(I \times \Omega)^{\prime}$. Then the system (17) has a unique solution in $B^{\alpha / 2}(I \times \Omega)$. Furthermore,

$$
\|u\|_{B^{\alpha / 2}(I \times \Omega)} \lesssim\|\bar{f}\|_{B^{\alpha / 2}(I \times \Omega)^{\prime}} .
$$

Proof. The existence and uniqueness of the solution of (17) is guaranteed by the well-known Lax-Milgram theorem. The continuity of the bilinear form $\mathscr{A}$ and the functional $\mathscr{F}$ is obvious. Now we need to prove the coercivity of $\mathscr{A}$ in the space $B^{\alpha / 2}(I \times \Omega)$. From the equivalence of ${ }^{l} H_{0}^{\alpha}(I \times \Omega)$, ${ }^{r} H_{0}^{\alpha}(I \times \Omega)$ and $H_{0}^{\alpha}(I \times \Omega)$, for all $u, v \in B^{\alpha / 2}(I \times \Omega)$, using the similar proof process in [32], we obtain

$$
\begin{aligned}
\mathscr{A}(v, v) \gtrsim & \left({ }_{0}^{R} D_{t}^{\alpha / 2} v,{ }_{0}^{R} D_{t}^{\alpha / 2} v\right)_{L_{2}(I \times \Omega)} \\
& +\sum_{i=1}^{s} a_{i}\left({ }_{0}^{R} D_{t}^{\alpha_{i} / 2} v,{ }_{0}^{R} D_{t}^{\alpha_{i} / 2} v\right)_{L_{2}(I \times \Omega)} \\
& +\left(\nabla_{x} u, \nabla_{x} v\right)_{L_{2}(I \times \Omega)} \gtrsim\|v\|_{B^{\alpha / 2}(I \times \Omega)}^{2} .
\end{aligned}
$$

Then we take $v=u$ in (17) to get $\|u\|_{B^{\alpha / 2}(I \times \Omega)}^{2} \lesssim(\bar{f}, u)_{L_{2}(I \times \Omega)}$ by the Schwarz inequality and the Poincaré inequality.

\section{Time Discretization and Convergence}

In this section, we consider DFBDM for the time discretization of (1)-(3), which is introduced in [33] for fractional ordinary differential equations. We can obtain the convergence order for the time discretization for the MT-FPDEs. Let $A=-\Delta_{x}, D(A)=H_{0}^{1}(\Omega) \cap H^{2}(\Omega)$. Let $u(t), f(t)$, and $u(0)$ denote the one-variable functions as $u(t, \cdot), f(t, \cdot)$, and $u(0, \cdot)$, respectively. Then (1) can be written in the abstract form, for $0<t<T, 0<\alpha_{s}<\cdots<\alpha_{1}<\alpha<1$, with initial value $u(0)=u_{0}$. Now we have

$$
{ }_{0}^{R} D_{t}^{\alpha}\left[u-u_{0}\right](t)+\sum_{i=1}^{s} a_{i 0}^{R} D_{t}^{\alpha_{i}}\left[u-u_{0}\right](t)+A u(t)=f(t) .
$$

Let $0=t_{0}<t_{1}<\cdots<t_{N}=T$ be a partition of $[0, T]$. Then, for fixed $t_{j}, j=1,2, \ldots, N$, we have

$$
{ }_{0}^{R} D_{t}^{\alpha}\left[u-u_{0}\right]\left(t_{j}\right)=\frac{t_{j}^{-\alpha}}{\Gamma(-\alpha)} \int_{0}^{1} g(\tau) \tau^{-1-\alpha} d \tau
$$

where $g(\tau)=u\left(t_{j}-t_{j} \tau\right)-u_{0}$. Here, the integral is a Hadamard finite-part integral in [33] and [34].

Now, for every $j$, we replace the integral by a firstdegree compound quadrature formula with equispaced nodes $0,(1 / j),(2 / j), \ldots, 1$ and obtain

$$
\int_{0}^{1} g(\tau) \tau^{-1-\alpha} d \tau=\sum_{k=0}^{j} \alpha_{k j}^{(\alpha)} g\left(\frac{k}{j}\right)+R_{j}^{(\alpha)}(g),
$$

where the weights $\alpha_{k j}^{(\alpha)}$ are

$$
\begin{aligned}
& \alpha(1-\alpha) j^{-\alpha} \alpha_{k j}^{(\alpha)} \\
& \quad= \begin{cases}-1, & \text { for } k=0, \\
2 k^{1-\alpha}-(k-1)^{1-\alpha}-(k+1)^{1-\alpha}, & \text { for } k=1,2, \ldots, j-1, \\
(\alpha-1) k^{-\alpha}-(k-1)^{1-\alpha}+k^{1-\alpha}, & \text { for } k=j,\end{cases}
\end{aligned}
$$

and the remainder term $R_{j}^{(\alpha)}(g)$ satisfies $\left\|R_{j}^{(\alpha)}(g)\right\| \leq$ $\gamma_{\alpha} j^{\alpha-2} \sup _{0 \leq t \leq T}\left\|g^{\prime \prime}(t)\right\|$, where $\gamma_{\alpha}>0$ is a constant.

Thus, for $\omega_{k j}^{(\alpha)}=j^{-\alpha} \alpha_{k j}^{(\alpha)} / \Gamma(-\alpha)$, we have

$$
\begin{aligned}
{ }_{0}^{R} D_{t}^{\alpha}\left[u-u_{0}\right]\left(t_{j}\right)= & \Delta t^{-\alpha} \sum_{k=0}^{j} \omega_{k j}^{(\alpha)}\left(u\left(t_{j}-t_{k}\right)-u(0)\right) \\
& +\frac{t_{j}^{-\alpha}}{\Gamma(-\alpha)} R_{j}^{(\alpha)}(g) .
\end{aligned}
$$


Let $t=t_{j}$, we can write (21) as

$$
\begin{aligned}
& \Delta t^{-\alpha} \sum_{k=0}^{j} \omega_{k j}^{(\alpha)}\left(u\left(t_{j}-t_{k}\right)-u(0)\right) \\
& +\sum_{i=1}^{s} a_{i} \Delta t^{-\alpha_{i}} \sum_{k=0}^{j} \omega_{k j}^{\left(\alpha_{i}\right)}\left(u\left(t_{j}-t_{k}\right)-u(0)\right)+A u\left(t_{j}\right) \\
& \quad=f\left(t_{j}\right)-\frac{t_{j}^{-\alpha}}{\Gamma(-\alpha)} R_{j}^{(\alpha)}(g)-\sum_{i=1}^{s} a_{i} \frac{t_{j}^{-\alpha_{i}}}{\Gamma\left(-\alpha_{i}\right)} R_{j}^{\left(\alpha_{i}\right)}(g), \\
& j=1,2,3, \ldots
\end{aligned}
$$

Denote $U^{j}$ as the approximation of $u\left(t_{j}\right)$ and $f_{j}=f\left(t_{j}\right)$. We obtain the following equation:

$$
\begin{aligned}
& \Delta t^{-\alpha} \sum_{k=0}^{j} \omega_{k j}^{(\alpha)}\left(U^{j-k}-U^{0}\right) \\
& +\sum_{i=1}^{s} a_{i} \Delta t^{-\alpha_{i}} \sum_{k=0}^{j} \omega_{k j}^{\left(\alpha_{i}\right)}\left(U^{j-k}-U^{0}\right)+A U^{j}=f_{j} .
\end{aligned}
$$

Lemma 3 (see [34]). For $0<\alpha<1$, let the sequence $\left\{d_{j}\right\}_{j=1,2, \ldots}$ be given by $d_{1}=1$ and $d_{j}=1+\alpha(1-\alpha) j^{-\alpha} \sum_{k=1}^{j-1} \alpha_{k j}^{(\alpha)} d_{j-k}$. Then, $1 \leq d_{j} \leq(\sin (\pi \alpha) / \pi \alpha(1-\alpha)) j^{\alpha}$, for $j=1,2,3, \ldots$

Let $e^{j}=u\left(t_{j}\right)-U^{j}$ denote the error in $t_{j}$. Then we have the following error estimate.

Theorem 4. Let $U^{j}$ and $u\left(t_{j}\right)$ be the solutions of (27) and (21), respectively. Then one has $\left\|U^{j}-u\left(t_{j}\right)\right\| \lesssim \Delta t^{2-\alpha}$.

Proof. Subtracting (27) from (26), we obtain the error equation

$$
\begin{gathered}
\Delta t^{-\alpha} \sum_{k=0}^{j} \omega_{k j}^{(\alpha)}\left(e^{j-k}-e^{0}\right)+\sum_{i=1}^{s} a_{i} \Delta t^{-\alpha_{i}} \sum_{k=0}^{j} \omega_{k j}^{\left(\alpha_{i}\right)}\left(e^{j-k}-e^{0}\right)+A e^{j} \\
\quad=-\frac{t_{j}^{-\alpha}}{\Gamma(-\alpha)} R_{j}^{(\alpha)}(g)-\sum_{i=1}^{s} a_{i} \frac{t_{j}^{-\alpha_{i}}}{\Gamma\left(-\alpha_{i}\right)} R_{j}^{\left(\alpha_{i}\right)}(g) .
\end{gathered}
$$

Note that $e^{0}=u(0)-U^{0}=0$. Denote

$$
\begin{gathered}
e^{j}=\left(\alpha_{0 j}^{(\alpha)}+\sum_{i=1}^{s} a_{i} \Delta t^{\alpha-\alpha_{i}} \frac{\Gamma(-\alpha)}{\Gamma\left(-\alpha_{i}\right)} \alpha_{0 j}^{\left(\alpha_{i}\right)}+A t^{\alpha} \Gamma(-\alpha)\right)^{-1} \\
\times\left(\sum_{k=1}^{j} \alpha_{k j}^{(\alpha)} e^{j-k}+\sum_{i=1}^{s} a_{i} \Delta t^{\alpha-\alpha_{i}} \frac{\Gamma(-\alpha)}{\Gamma\left(-\alpha_{i}\right)} \sum_{k=1}^{j} \alpha_{k j}^{\left(\alpha_{i}\right)} e^{j-k}\right. \\
\left.-R_{j}^{(\alpha)}(g)-\sum_{i=1}^{s} a_{i} \frac{\Gamma(-\alpha)}{\Gamma\left(-\alpha_{i}\right)} t_{j}^{\alpha-\alpha_{i}} R_{j}^{\left(\alpha_{i}\right)}(g)\right)
\end{gathered}
$$

Let $\|\cdot\|$ denote the $L_{2}$-norm, then we have

$$
\begin{aligned}
\left\|e^{j}\right\| \leq & \left\|\left(\alpha_{0 j}^{(\alpha)}+\sum_{i=1}^{s} a_{i} \Delta t^{\alpha-\alpha_{i}} \frac{\Gamma(-\alpha)}{\Gamma\left(-\alpha_{i}\right)} \alpha_{0 j}^{\left(\alpha_{i}\right)}+A t^{\alpha} \Gamma(-\alpha)\right)^{-1}\right\| \\
& \times\left(\sum_{k=1}^{j} \alpha_{k j}^{(\alpha)}\left\|e^{j-k}\right\|+\sum_{i=1}^{s} a_{i} \Delta t^{\alpha-\alpha_{i}} \frac{\Gamma(-\alpha)}{\Gamma\left(-\alpha_{i}\right)} \sum_{k=1}^{j} \alpha_{k j}^{\left(\alpha_{i}\right)}\left\|e^{j-k}\right\|\right. \\
& \left.+\left\|R_{j}^{(\alpha)}(g)\right\|+\sum_{i=1}^{s} a_{i} \frac{\Gamma(-\alpha)}{\Gamma\left(-\alpha_{i}\right)} t_{j}^{\alpha-\alpha_{i}}\left\|R_{j}^{\left(\alpha_{i}\right)}(g)\right\|\right) .
\end{aligned}
$$

Note that $A$ is a positive definite elliptic operator with all of eigenvalues $\lambda>0$. Since $\alpha_{0 j}^{(\alpha)}<0$ and $\Gamma(-\alpha)<0$, we have

$$
\begin{aligned}
& \left\|\left(\alpha_{0 j}^{(\alpha)}+\sum_{i=1}^{s} a_{i} \Delta t^{\alpha-\alpha_{i}} \frac{\Gamma(-\alpha)}{\Gamma\left(-\alpha_{i}\right)} \alpha_{0 j}^{\left(\alpha_{i}\right)}+A t^{\alpha} \Gamma(-\alpha)\right)^{-1}\right\| \\
& =\sup _{\lambda>0}\left\|\left(\alpha_{0 j}^{(\alpha)}+\sum_{i=1}^{s} a_{i} \Delta t^{\alpha-\alpha_{i}} \frac{\Gamma(-\alpha)}{\Gamma\left(-\alpha_{i}\right)} \alpha_{0 j}^{\left(\alpha_{i}\right)}+\lambda t^{\alpha} \Gamma(-\alpha)\right)^{-1}\right\| \\
& \lesssim\left(-\alpha_{0 j}^{(\alpha)}-\sum_{i=1}^{s} a_{i} \Delta t^{\alpha-\alpha_{i}} \frac{\Gamma(-\alpha)}{\Gamma\left(-\alpha_{i}\right)} \alpha_{0 j}^{\left(\alpha_{i}\right)}\right)^{-1} .
\end{aligned}
$$

Hence,

$$
\begin{aligned}
\left\|e^{j}\right\| \leq & \alpha(1-\alpha) j^{-\alpha} \sum_{k=1}^{j} \alpha_{k j}^{(\alpha)}\left\|e^{j-k}\right\| \\
& +\sum_{i=1}^{s} \alpha_{i}\left(1-\alpha_{i}\right) j^{-\alpha_{i}} \sum_{k=1}^{j} \alpha_{k j}^{\left(\alpha_{i}\right)}\left\|e^{j-k}\right\| \\
& +\alpha(1-\alpha) \gamma_{\alpha} n^{-2} \sup _{0 \leq t \leq T}\left\|u^{\prime \prime}\right\| \\
& +\sum_{i=1}^{s} \alpha_{i}\left(1-\alpha_{i}\right) \gamma_{\alpha_{i}} n^{-2} \sup _{0 \leq t \leq T}\left\|u^{\prime \prime}\right\| .
\end{aligned}
$$

Denote $d_{1}=1$ and

$$
\begin{gathered}
d_{j}=1+\alpha(1-\alpha) j^{-\alpha} \sum_{k=1}^{j-1} \alpha_{k j}^{(\alpha)} d_{j-k}, \quad j=2,3, \ldots, n, \\
d_{j}^{i}=1+\alpha_{i}\left(1-\alpha_{i}\right) j^{-\alpha_{i}} \sum_{k=1}^{j-1} \alpha_{k j}^{\left(\alpha_{i}\right)} d_{j-k}, \quad j=2,3, \ldots, n,
\end{gathered}
$$


where $i=1,2, \ldots, s$. By induction and Lemma 3, then we have

$$
\begin{aligned}
\left\|e^{j}\right\| \leq & \alpha(1-\alpha) n^{-2} \sup _{0 \leq t \leq T}\left\|u^{\prime \prime}(t)\right\| \cdot d_{j} \\
& +\sum_{i=1}^{s} \alpha_{i}\left(1-\alpha_{i}\right) n^{-2} \sup _{0 \leq t \leq T}\left\|u^{\prime \prime}(t)\right\| \cdot d_{j}^{i} \\
\leq & n^{-2} \sup _{0 \leq t \leq T}\left\|u^{\prime \prime}(t)\right\| \frac{\sin (\pi \alpha)}{\pi} j^{\alpha} \\
& +\sum_{i=1}^{s} n^{-2} \sup _{0 \leq t \leq T}\left\|u^{\prime \prime}(t)\right\| \frac{\sin \left(\pi \alpha_{i}\right)}{\pi} j^{\alpha_{i}} \\
\leq & \Delta t^{2-\alpha}+\sum_{i=1}^{s} \Delta t^{2-\alpha_{i}} .
\end{aligned}
$$

\section{Space Discretization and Convergence}

In this section, we will consider the space discretization for MT-FPDEs (1) and show the complete process and details of numerical scheme. The variational form of (1) is to find $u(t, \cdot) \in H_{0}^{1}(\Omega)$, such that, for all $v \in H_{0}^{1}(\Omega)$,

$$
\begin{aligned}
& \left({ }_{0}^{R} D_{t}^{\alpha} u(t, x), v\right)_{L_{2}(\Omega)}+\sum_{i=1}^{s} a_{i}\left({ }_{0}^{R} D_{t}^{\alpha_{i}} u(t, x), v\right)_{L_{2}(\Omega)} \\
& +\left(\nabla_{x} u, \nabla_{x} v\right)=(\bar{f}(t, x), v)_{L_{2}(\Omega)} .
\end{aligned}
$$

Let $h$ denote the maximal length of intervals in $\Omega$ and let $r$ be any nonnegative integer. We denote the norm in $H^{r}(\Omega)$ by $\|\cdot\|_{H^{r}(\Omega)}$. Let $S_{h} \subset H_{0}^{r}$ be a family of finite element spaces with the accuracy of order $r \geq 2$, that is, $S_{h}$ consists of continuous functions on the closure $\bar{\Omega}$ of $\Omega$ which are polynomials of degree at most $r-1$ in each interval and which vanish outside $\Omega_{h}$, such that for small $h, v \in H^{b}(\Omega) \cap H_{0}^{1}(\Omega)$,

$$
\begin{array}{r}
\inf _{\chi \in S_{h}}\left(\|v-\chi\|_{L_{2}(\Omega)}+h\left\|\nabla_{x}(v-\chi)\right\|_{L_{2}(\Omega)}\right) \leq C h^{b}\|v\|_{H^{b}(\Omega)}, \\
1 \leq b \leq r .
\end{array}
$$

The semidiscrete problem of (1) is to find the approximate solution $u_{h}(t)=u_{h}(t, \cdot) \in S_{h}$ and $\bar{f}(t)=\bar{f}(t, \cdot)$ for each $t$ such that

$$
\begin{aligned}
& \left({ }_{0}^{R} D_{t}^{\alpha} u_{h}(t), \chi\right)_{L_{2}(\Omega)}+\sum_{i=1}^{s} a_{i}\left({ }_{0}^{R} D_{t}^{\alpha_{i}} u_{h}(t), \chi\right)_{L_{2}(\Omega)} \\
& \quad+\left(\nabla_{x} u_{h}(t), \nabla_{x} \chi\right)_{L_{2}(\Omega)}=(\bar{f}(t), \chi)_{L_{2}(\Omega)}, \quad \forall \chi \in S_{h} .
\end{aligned}
$$

Let $U^{N}=u_{h}\left(t_{N}, x\right)$. After the time discretization, we have

$$
\begin{gathered}
\Delta t^{-\alpha} \sum_{k=0}^{N} \omega_{k N}^{(\alpha)}\left(U^{N}, \chi\right)_{L_{2}(\Omega)}+\sum_{i=1}^{s} a_{i} \Delta t^{-\alpha_{i}} \sum_{k=0}^{N} \omega_{k N}^{\left(\alpha_{i}\right)}\left(U^{N}, \chi\right)_{L_{2}(\Omega)} \\
+\left(\nabla_{x} U^{N}, \nabla_{x} \chi\right)_{L_{2}(\Omega)}=(\bar{f}(t), \chi)_{L_{2}(\Omega)}, \quad \forall \chi \in S_{h} .
\end{gathered}
$$

In terms of the basis $\left\{\psi_{m}\right\}_{m=1}^{M-1} \subseteq S_{h}$, choosing $\chi=\psi_{m}$, writing

$$
u_{h}\left(t_{N}, x\right)=\sum_{j=1}^{M-1} U_{j}^{N} \psi_{j}(x),
$$

and inserting it into (38), one obtains

$$
\begin{aligned}
& \sum_{j=1}^{M-1} \Delta t^{-\alpha} \sum_{k=1}^{N} \omega_{k N}^{(\alpha)} U_{j}^{N-k}\left(\psi_{j}, \psi_{m}\right)_{L_{2}(\Omega)} \\
& +\sum_{i=1}^{s} a_{i} \sum_{j=1}^{M-1} \Delta t^{-\alpha_{i}} \sum_{k=1}^{N} \omega_{k N}^{\left(\alpha_{i}\right)} U_{j}^{N-k}\left(\psi_{j}, \psi_{m}\right)_{L_{2}(\Omega)} \\
& +\sum_{j=1}^{M-1} U_{j}^{N}\left(\nabla_{x} \psi_{j}, \nabla_{x} \psi_{m}\right)_{L_{2}(\Omega)}=\left(\bar{f}, \psi_{m}\right)_{L_{2}(\Omega)}, \\
& m=1,2, \ldots, M-1 .
\end{aligned}
$$

Let $U_{N}=\left(U_{1}^{N}, U_{2}^{N}, \ldots, U_{M-1}^{N}\right)^{T}$. From (40), we obtain a vector equation

$$
\begin{aligned}
& \Psi_{1}\left(\Delta t^{-\alpha} \omega_{0 N}^{(\alpha)} U_{N}+\sum_{i=1}^{s} a_{i} \Delta t^{-\alpha_{i}} \omega_{0 N}^{\left(\alpha_{i}\right)} U_{N}\right)+\Psi_{2} U_{N} \\
& \quad=\Psi_{1}\left(F_{N}-\Delta t^{-\alpha} \sum_{k=1}^{N} \omega_{k N}^{(\alpha)} U_{N-k}-\sum_{i=1}^{s} a_{i} \Delta t^{-\alpha_{i}} \sum_{k=1}^{N} \omega_{k N}^{\left(\alpha_{i}\right)} U_{N-k}\right),
\end{aligned}
$$

where initial condition is $U_{0}=u(0, x), \Psi_{1}:=\left\{\left(\psi_{j}\right.\right.$, $\left.\left.\psi_{m}\right)_{L_{2}(\Omega)}\right\}_{j, m=1}^{M-1}$ is the mass matrix, $\Psi_{2}$ is stiffness matrix as $\Psi_{2}:=\left\{\left(\nabla_{x} \psi_{j}, \nabla_{x} \psi_{m}\right)_{L_{2}(\Omega)}\right\}_{j, m=1}^{M-1}$, and $F_{N}:=\left(\bar{f}_{1}, \ldots, \bar{f}_{M-1}\right)^{T}$ is a vector valued function. Then, we can obtain the solution $U_{N}$ at $t=t_{N}$.

Let $R_{h}: H^{1}(\Omega) \rightarrow S_{h}$ be the elliptic projection, defined by $\left(\nabla_{x} R_{h} \mathcal{u}, \nabla_{x} \chi\right)_{L_{2}(\Omega)}=\left(\nabla_{x} \mathcal{u}, \nabla_{x} \chi\right)_{L_{2}(\Omega)}$, for all $\chi \in S_{h}$.

Lemma 5 (see [35]). Assume that (36) holds, then with $R_{h}$ and $v \in H^{b}(\Omega) \cap H_{0}^{1}(\Omega)$, we have $\left\|R_{h} v-v\right\|_{L_{2}(\Omega)}+h \| \nabla_{x}\left(R_{h} v-\right.$ $v)\left\|_{L_{2}(\Omega)} \leq C h^{b}\right\| v \|_{H^{b}(\Omega)}$ for $1 \leq b \leq r$.

In virtue of the standard error estimate for the FEM of MT-FPDEs, one has the following theorem which can be proved easily by Lemma 5 and the similar proof in [35].

Theorem 6. For $0<\alpha_{s}<\cdots<\alpha_{1}<\alpha<1$, let $u_{h} \in S_{h}$ and $u(t, \cdot) \in H_{0}^{1}(\Omega)$ be, respectively, the solutions of (37) and (1), then $\left\|u-u_{h}\right\|_{L_{2}(\Omega)} \leqslant h^{2}\|u\|_{L_{2}(\Omega)}$.

\section{Stability of the Numerical Method}

In this section, we analyze the stability of the FEM for MT-FPEDs (1)-(3). Now we do some preparations before proving the stability of the method. Based on the definition of coefficients $\omega_{k j}^{(\alpha)}$ in Section 3, we can obtain the following lemma easily. 
Lemma 7. For $0<\alpha<1$, the coefficients $\omega_{k j}^{(\alpha)},(k=1, \ldots, j)$ satisfy the following properties:

(i) $\omega_{0 j}^{(\alpha)}>0$ and $\omega_{k j}^{(\alpha)}<0$ for $k=1,2, \ldots, j$,

(ii) $\Gamma(2-\alpha) \sum_{k=1}^{j} \omega_{k j}^{(\alpha)}=(1-\alpha) j^{-\alpha}+1$.

Now we report the stability theorem of this FEM for MTFPDEs in this section as follows.

Theorem 8. The FEM defined as in (38) is unconditionally stable.

Proof. In (38), let $\chi(\cdot)=U^{j}(\cdot)$ at $t=t_{j}$ and the right hand $\bar{f}=0$. We have

$$
\begin{aligned}
& \Delta t^{-\alpha}\left\{\frac{1}{\Gamma(2-\alpha)}\left(U^{j}, U^{j}\right)_{L_{2}(\Omega)}+\sum_{k=1}^{j-1} \omega_{k j}^{(\alpha)}\left(U^{j-k}, U^{j}\right)_{L_{2}(\Omega)}\right. \\
& \left.+\omega_{j j}^{(\alpha)}\left(U^{0}, U^{j}\right)_{L_{2}(\Omega)}\right\} \\
& +\sum_{i=1}^{s} a_{i} \Delta t^{-\alpha_{i}}\left\{\frac{1}{\Gamma\left(2-\alpha_{i}\right)}\left(U^{j}, U^{j}\right)_{L_{2}(\Omega)}\right. \\
& \left.\quad+\sum_{k=1}^{j-1} \omega_{k j}^{\left(\alpha_{i}\right)}\left(U^{j-k}, U^{j}\right)_{L_{2}(\Omega)}+\omega_{j j}^{\left(\alpha_{i}\right)}\left(U^{0}, U^{j}\right)_{L_{2}(\Omega)}\right\} \\
& +\left(\nabla_{x} U^{j}, \nabla_{x} U^{j}\right)_{L_{2}(\Omega)}=0 .
\end{aligned}
$$

Using, Cauchy-Schwarz inequality, $\pm\left(U^{j-k}, U^{j}\right) \leq$ $(1 / 2)\left(\left\|U^{j-k}\right\|_{L_{2}(\Omega)}^{2}+\left\|U^{j}\right\|_{L_{2}(\Omega)}^{2}\right)$ for $k=0,1,2, \ldots, N$ and Lemma 7 , we get

$$
\begin{aligned}
& \left(\frac{\Delta t^{-\alpha}}{2 \Gamma(2-\alpha)}\left(1+(1-\alpha) j^{-\alpha}\right)\right. \\
& \left.+\sum_{i=1}^{s} a_{i} \frac{\Delta t^{-\alpha_{i}}}{2 \Gamma\left(2-\alpha_{i}\right)}\left(1+\left(1-\alpha_{i}\right) j^{-\alpha_{i}}\right)\right)\left\|U^{j}\right\|_{L_{2}(\Omega)}^{2}+\left\|\nabla_{x} U^{j}\right\|_{L_{2}(\Omega)}^{2} \\
& \leq \frac{\Delta t^{-\alpha}}{2}\left[-\sum_{k=1}^{j-1} \omega_{k j}^{(\alpha)}\left\|U^{j-k}\right\|_{L_{2}(\Omega)}^{2}-\omega_{j j}^{(\alpha)}\left\|U^{0}\right\|_{L_{2}(\Omega)}^{2}\right] \\
& \quad+\sum_{i=1}^{s} a_{i} \frac{\Delta t^{-\alpha_{i}}}{2}\left[-\sum_{k=1}^{j-1} \omega_{k j}^{\left(\alpha_{i}\right)}\left\|U^{j-k}\right\|_{L_{2}(\Omega)}^{2}-\omega_{j j}^{\left(\alpha_{i}\right)}\left\|U^{0}\right\|_{L_{2}(\Omega)}^{2}\right] .
\end{aligned}
$$

We prove the stability of (37) by induction. Since when $j=1$, we have

$$
\begin{aligned}
& \left(\frac{\Delta t^{-\alpha}}{2 \Gamma(2-\alpha)}(1+(1-\alpha))\right. \\
& \left.+\sum_{i=1}^{s} a_{i} \frac{\Delta t^{-\alpha_{i}}}{2 \Gamma\left(2-\alpha_{i}\right)}\left(1+\left(1-\alpha_{i}\right)\right)\right)\left\|U^{1}\right\|_{L_{2}(\Omega)}^{2} \\
& \leq\left(\frac{\Delta t^{-\alpha}}{2 \Gamma(2-\alpha)}(1-(1-\alpha))\right. \\
& \left.\quad+\sum_{i=1}^{s} a_{i} \frac{\Delta t^{-\alpha_{i}}}{2 \Gamma\left(2-\alpha_{i}\right)}\left(1-\left(1-\alpha_{i}\right)\right)\right)\left\|U^{0}\right\|_{L_{2}(\Omega)} .
\end{aligned}
$$

The induction basis $\left\|U^{1}\right\|_{L_{2}(\Omega)} \leq\left\|U^{0}\right\|_{L_{2}(\Omega)}$ is presupposed. For the induction step, we have $\left\|U^{j}\right\|_{L_{2}(\Omega)} \leq\left\|U^{j-1}\right\|_{L_{2}(\Omega)} \leq$ $\cdots \leq\left\|U^{0}\right\|_{L_{2}(\Omega)}$. Then using this result, by Lemma 7 , we obtain

$$
\begin{aligned}
& \left(\frac{\Delta t^{-\alpha}\left(1+(1-\alpha)(j+1)^{-\alpha}\right)}{2 \Gamma(2-\alpha)}\right. \\
& \left.+\sum_{i=1}^{s} a_{i} \frac{\Delta t^{-\alpha_{i}}}{2 \Gamma\left(2-\alpha_{i}\right)}\left(1+\left(1-\alpha_{i}\right)(j+1)^{-\alpha_{i}}\right)\right)\left\|U^{j+1}\right\|_{L_{2}(\Omega)}^{2} \\
& \leq\left(\frac{\Delta t^{-\alpha}\left(1-(1-\alpha)(j+1)^{-\alpha}\right)}{2 \Gamma(2-\alpha)}\right. \\
& \left.\quad+\sum_{i=1}^{s} a_{i} \frac{\Delta t^{-\alpha_{i}}}{2 \Gamma\left(2-\alpha_{i}\right)}\left(1-\left(1-\alpha_{i}\right)(j+1)^{-\alpha_{i}}\right)\right)\left\|U^{0}\right\|_{L_{2}(\Omega)}^{2}
\end{aligned}
$$

Here $0<1-\alpha<1$. After squaring at both sides of the above inequality, we obtain $\left\|U^{j+1}\right\|_{L_{2}(\Omega)} \leq\left\|U^{0}\right\|_{L_{2}(\Omega)}$.

\section{Numerical Experiments}

In this section, we present the numerical examples of MTFPDEs to demonstrate the effectiveness of our theoretical analysis. The main purpose is to check the convergence behavior of numerical solutions with respect to $\Delta t$ and $\Delta x$, which have been shown in Theorem 4 and Theorem 6 . It is noted that the method in [29] is a special case of the method in our paper for fractional partial differential equation with single fractional order. So, we just need to compare FEM in our paper with other existing methods in $[8,28]$.

Example 9. For $t \in[0, T], x \in(0,1)$, consider the MT-FPDEs with two variables as follows:

$$
\begin{gathered}
{ }_{0}^{C} D_{t}^{\alpha} u(t, x)+{ }_{0}^{C} D_{t}^{\beta} u(t, x)-\partial_{x}^{2} u(t, x)=f(t, x), \\
u(0, x)=0, \quad x \in(0,1), \\
u(t, 0)=u(t, 1)=0, \quad t \in[0, T],
\end{gathered}
$$




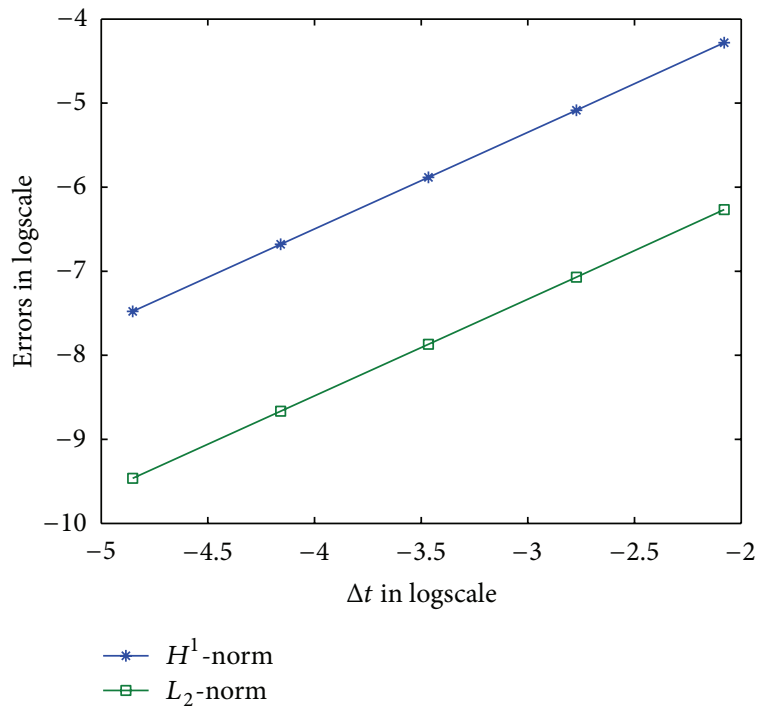

(a)

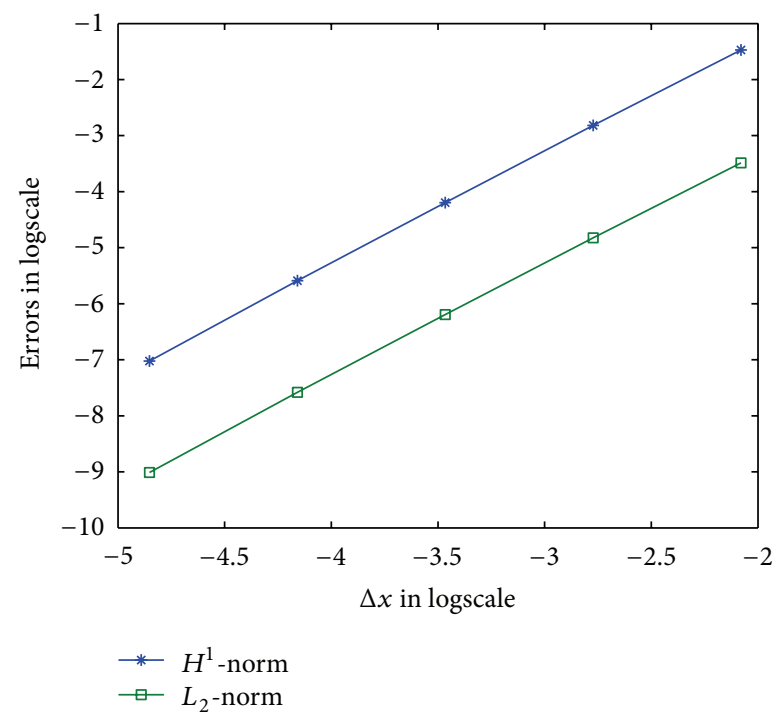

(b)

FIgURE 1: $H^{1}$-norm and $L_{2}$-norm of errors for (46) with $\alpha=0.9, \beta=0.5, \Delta x=0.001$ (a), and $\Delta t=0.001$ (b).

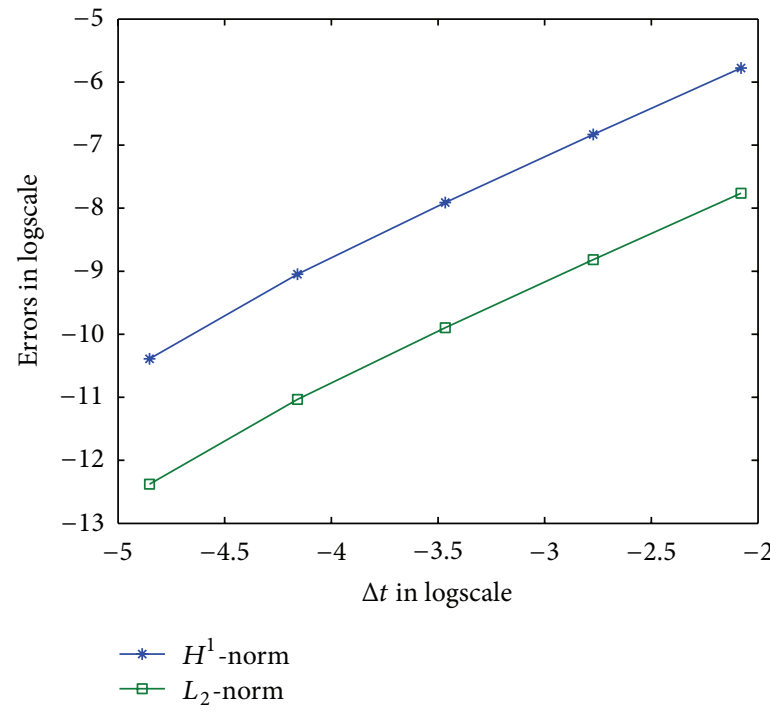

(a)

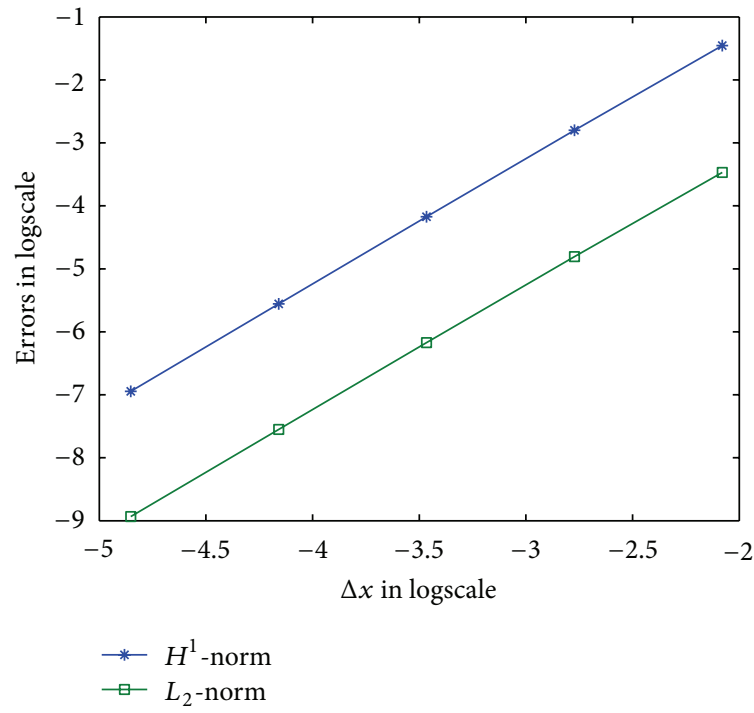

(b)

FIGURE 2: $H^{1}$-norm and $L_{2}$-norm of errors for (46) with $\alpha=0.5, \beta=0.25, \Delta x=0.001$ (a), and $\Delta t=0.001$ (b).

where the right-side function $f(t, x)=\left(2 t^{2-\alpha} / \Gamma(3-\right.$ $\alpha)) \sin (2 \pi x)+\left(2 t^{2-\beta} / \Gamma(3-\beta)\right) \sin (2 \pi x)+4 \pi^{2} \sin (2 \pi x) t^{2}$. The exact solution is $u(t, x)=t^{2} \sin (2 \pi x)$.

We use this example to check the convergence rate (c. rate) and CPU time (CPUT) of numerical solutions with respect to the fractional orders $\alpha$ and $\beta$.

In the first test, we fix $T=1, \alpha=0.9$ and $\beta=0.5$ and choose $\Delta x=0.001$ which is small enough such that the space discretization errors are negligible as compared with the time errors. Choosing $\Delta t=1 / 2^{i}(i=2,4, \ldots, 7)$, we report that the convergence rate of FDM in time is nearly 1.15 in Table 1 , which matches well with the result of Theorem 4 . On the other hand, Table 2 shows that an approximate convergence rate is 2 , by fixing $\Delta t=0.001$ and choosing $\Delta x=1 / 2^{i}(i=$ $2, \ldots, 6)$, which matches well with the result of Theorem 6 . In the second test, we give the convergence rate when $\alpha=0.5$, $\beta=0.25$ for $\Delta t$ in Table 3 , and $\Delta x$ in Table 4 , respectively. We also report the $L_{2}$-norm and $H^{1}$-norm of errors in Figures 1 and 2 , respectively.

Fixing $\Delta x=0.001, \alpha=0.9$, and $\beta=0.3$ in (46), we compare the error and CPUT calculated by the FEM in this paper with the FDM in [8] and the FPCM in [8]. From Table 5, it can be seen that the FEM in this paper is computationally effective. 
TABLE 1: Convergence rate in time for (46) with $\alpha=0.9$ and $\beta=0.5$.

\begin{tabular}{lccccc}
\hline$\Delta x$ & $\Delta t$ & $H^{1}$-norm & $L_{2}$-norm & c. rate & CPUT (seconds) \\
\hline 0.001 & $1 / 4$ & $1.3815 \times 10^{-2}$ & $1.8960 \times 10^{-3}$ & & 0.214 \\
0.001 & $1 / 16$ & $6.1890 \times 10^{-3}$ & $8.4939 \times 10^{-4}$ & 1.1585 & 0.357 \\
0.001 & $1 / 32$ & $2.7858 \times 10^{-3}$ & $3.8234 \times 10^{-4}$ & 1.1516 & 0.736 \\
0.001 & $1 / 64$ & $1.2571 \times 10^{-3}$ & $1.7252 \times 10^{-4}$ & 1.1481 & 1.438 \\
0.001 & $1 / 128$ & $5.6567 \times 10^{-4}$ & $7.7635 \times 10^{-5}$ & 1.1520 & 2.922 \\
\hline
\end{tabular}

TABLE 2: Convergence rate in space for (46) with $\alpha=0.9$ and $\beta=0.5$.

\begin{tabular}{lccccc}
\hline$\Delta t$ & $\Delta x$ & $H^{1}$-norm & $L_{2}$-norm & c. rate & CPUT (seconds) \\
\hline 0.001 & $1 / 4$ & 0.2294 & $3.0611 \times 10^{-2}$ & & 20.35 \\
0.001 & $1 / 16$ & $5.9763 \times 10^{-2}$ & $8.0381 \times 10^{-3}$ & 1.9291 & 21.85 \\
0.001 & $1 / 32$ & $1.5067 \times 10^{-2}$ & $2.0442 \times 10^{-3}$ & 1.9753 & 26.68 \\
0.001 & $1 / 64$ & $3.7356 \times 10^{-3}$ & $5.0966 \times 10^{-4}$ & 2.0039 & 32.72 \\
0.001 & $1 / 128$ & $8.9129 \times 10^{-4}$ & $1.2198 \times 10^{-4}$ & 2.0629 & 41.03 \\
\hline
\end{tabular}

TABLE 3: Convergence rate in time for (46) with $\alpha=0.5$ and $\beta=0.25$.

\begin{tabular}{lccccc}
\hline$\Delta x$ & $\Delta t$ & $H^{1}$-norm & $L_{2}$-norm & c. rate & CPUT (seconds) \\
\hline 0.001 & $1 / 4$ & $3.0985 \times 10^{-3}$ & $4.2525 \times 10^{-4}$ & 1.5221 & 0.218 \\
0.001 & $1 / 16$ & $1.0789 \times 10^{-3}$ & $1.4807 \times 10^{-4}$ & 1.5556 & 0.413 \\
0.001 & $1 / 32$ & $3.6702 \times 10^{-4}$ & $5.0372 \times 10^{-5}$ & 1.6406 & 0.921 \\
0.001 & $1 / 64$ & $1.1772 \times 10^{-4}$ & $1.6156 \times 10^{-5}$ & 1.6388 & 3.783 \\
0.001 & $1 / 128$ & $3.0704 \times 10^{-5}$ & $4.2139 \times 10^{-6}$ & & 1.855 \\
\hline
\end{tabular}

TABLE 4: Convergence rate in space for (46) with $\alpha=0.5$ and $\beta=0.25$.

\begin{tabular}{lccccc}
\hline$\Delta t$ & $\Delta x$ & $H^{1}$-norm & $L_{2}$-norm & c. rate & CPUT (seconds) \\
\hline 0.001 & $1 / 4$ & $2.3325 \times 10^{-1}$ & $3.1119 \times 10^{-2}$ & & 23.73 \\
0.001 & $1 / 16$ & $6.0836 \times 10^{-2}$ & $8.1823 \times 10^{-3}$ & 1.9272 & 26.29 \\
0.001 & $1 / 32$ & $1.5382 \times 10^{-2}$ & $2.0870 \times 10^{-3}$ & 1.9711 & 33.49 \\
0.001 & $1 / 64$ & $3.8569 \times 10^{-3}$ & $5.2622 \times 10^{-4}$ & 1.9877 & 41.68 \\
0.001 & $1 / 128$ & $9.6378 \times 10^{-4}$ & $1.3189 \times 10^{-4}$ & 1.9963 & 55.24 \\
\hline
\end{tabular}

TABLE 5: Comparison of error and CPUT for (46) with $\alpha=0.9$ and $\beta=0.3$.

\begin{tabular}{lccccccc}
\hline$\Delta x$ & $\Delta t$ & \multicolumn{2}{c}{ FEM } & \multicolumn{2}{c}{ FDM [8] } & \multicolumn{2}{c}{ FPCM [8] } \\
& & Error & CPUT & Error & CPUT & Error & CPUT \\
\hline 0.001 & $1 / 4$ & $3.7056 \times 10^{-3}$ & 0.238 & $5.8723 \times 10^{-3}$ & 0.897 & $2.2027 \times 10^{-2}$ & 6.16 \\
0.001 & $1 / 8$ & $1.6794 \times 10^{-3}$ & 0.481 & $2.6751 \times 10^{-3}$ & 1.837 & $8.7467 \times 10^{-3}$ & 16.63 \\
0.001 & $1 / 16$ & $7.6528 \times 10^{-4}$ & 0.962 & $1.2159 \times 10^{-3}$ & 3.512 & $3.4693 \times 10^{-3}$ & 30.11 \\
0.001 & $1 / 32$ & $3.5009 \times 10^{-4}$ & 1.335 & $5.5190 \times 10^{-4}$ & 7.001 & $1.3765 \times 10^{-3}$ & 52.71 \\
0.001 & $1 / 64$ & $1.6027 \times 10^{-4}$ & 2.703 & $2.4997 \times 10^{-4}$ & 14.45 & $5.4564 \times 10^{-4}$ & 106.49 \\
\hline
\end{tabular}

TABLE 6: Comparison of error, convergence rate, and CPUT for (47) with $\alpha=0.5$ and $\beta=0.3$.

\begin{tabular}{|c|c|c|c|c|c|c|}
\hline \multirow{2}{*}{$\Delta t$} & \multicolumn{3}{|c|}{ DFBDM (Section 3) } & \multicolumn{3}{|c|}{ FEM2 [28] } \\
\hline & Error & c. rate & CPUT & Error & c. rate & CPUT \\
\hline $1 / 4$ & $9.1975 \times 10^{-4}$ & & 0.000864 & $3.6606 \times 10^{-3}$ & & 0.001862 \\
\hline $1 / 8$ & $3.3037 \times 10^{-4}$ & 1.4772 & 0.001986 & $7.8173 \times 10^{-3}$ & 2.2274 & 0.004902 \\
\hline $1 / 16$ & $1.1375 \times 10^{-4}$ & 1.5382 & 0.004649 & $1.6210 \times 10^{-4}$ & 2.2697 & 0.051816 \\
\hline $1 / 32$ & $3.5112 \times 10^{-5}$ & 1.6958 & 0.012112 & $3.2629 \times 10^{-4}$ & 2.3127 & 0.518130 \\
\hline
\end{tabular}


Example 10. Consider the following multierm fractional differential problem:

$$
{ }_{0}^{C} D_{t}^{\alpha} u(t)+t^{-0.2}{ }_{0}^{C} D_{t}^{\beta} u(t)=f(t), \quad u(0)=0,
$$

where $f(t)=\left(4 t^{1.5} / \Gamma(2.5)\right)+\left(12 t^{2} / \Gamma(3.5)\right)$. For $\alpha=0.5$ and $\beta=0.3$, the exact solution is $u(t)=t^{2}+t^{2.5}$.

For the problem (47), our method in this paper is just the DFBDM in Section 3. Therefore, we only need to compare M1 with the FEM in [28] (FEM2). In Table 6, although the convergence rate of FEM2 is higher than that of DFBDM, the error and CPUT of DFBDM are smaller than those of FEM2.

\section{Acknowledgments}

The authors are grateful to the referees for their valuable comments. This work is supported by the National Natural Science Foundation of China (11101109 and 11271102), the Natural Science Foundation of Hei-Long-Jiang Province of China (A201107), and SRF for ROCS, SEM.

\section{References}

[1] C. Li, Z. Zhao, and Y. Chen, "Numerical approximation and error estimates of a time fractional order diffusion equation," in Proceedings of the ASME International Design Engineering Technical Conference and Computers and Information in Engineering Conference (IDETC/CIE '09), San Diego, Calif, USA, 2009.

[2] Y. Jiang and J. Ma, "High-order finite element methods for timefractional partial differential equations," Journal of Computational and Applied Mathematics, vol. 235, no. 11, pp. 3285-3290, 2011.

[3] X. J. Li and C. J. Xu, "Existence and uniqueness of the weak solution of the space-time fractional diffusion equation and a spectral method approximation," Communications in Computational Physics, vol. 8, no. 5, pp. 1016-1051, 2010.

[4] K. Diethelm and N. J. Ford, "Numerical solution of the BagleyTorvik equation," Numerical Mathematics, vol. 42, no. 3, pp. 490-507, 2002.

[5] K. Diethelm and N. J. Ford, "Multi-order fractional differential equations and their numerical solution," Applied Mathematics and Computation, vol. 154, no. 3, pp. 621-640, 2004.

[6] V. Daftardar-Gejji and S. Bhalekar, "Boundary value problems for multi-term fractional differential equations," Journal of Mathematical Analysis and Applications, vol. 345, no. 2, pp. 754765, 2008.

[7] H. Jiang, F. Liu, I. Turner, and K. Burrage, "Analytical solutions for the multi-term time-space Caputo-Riesz fractional advection-diffusion equations on a finite domain," Journal of Mathematical Analysis and Applications, vol. 389, no. 2, pp. 11171127, 2012.

[8] F. Liu, M. M. Meerschaert, R. J. McGough, P. Zhuang, and Q. Liu, "Numerical methods for solving the multi-term timefractional wave-diffusion equation," Fractional Calculus and Applied Analysis, vol. 16, no. 1, pp. 9-25, 2013.

[9] G. Psihoyios and T. E. Simos, "Trigonometrically fitted predictor-corrector methods for IVPs with oscillating solutions," Journal of Computational and Applied Mathematics, vol. 158, no. 1, pp. 135-144, 2003.
[10] T. E. Simos, I. T. Famelis, and C. Tsitouras, "Zero dissipative, explicit Numerov-type methods for second order IVPs with oscillating solutions," Numerical Algorithms, vol. 34, no. 1, pp. 27-40, 2003.

[11] T. E. Simos, "Dissipative trigonometrically-fitted methods for linear second-order IVPs with oscillating solution," Applied Mathematics Letters, vol. 17, no. 5, pp. 601-607, 2004.

[12] G. Psihoyios and T. E. Simos, "A fourth algebraic order trigonometrically fitted predictor-corrector scheme for IVPs with oscillating solutions," Journal of Computational and Applied Mathematics, vol. 175, no. 1, pp. 137-147, 2005.

[13] S. Stavroyiannis and T. E. Simos, "Optimization as a function of the phase-lag order of nonlinear explicit two-step P.-stable method for linear periodic IVPs," Applied Numerical Mathematics, vol. 59, no. 10, pp. 2467-2474, 2009.

[14] G. A. Panopoulos and T. E. Simos, "An optimized symmetric 8-Step semi-embedded predictor-corrector method for IVPs with oscillating solutions," Applied Mathematics \& Information Sciences, vol. 7, no. 1, pp. 73-80, 2013.

[15] A. Konguetsof and T. E. Simos, "A generator of hybrid symmetric four-step methods for the numerical solution of the Schrödinger equation," Journal of Computational and Applied Mathematics, vol. 158, no. 1, pp. 93-106, 2003.

[16] Z. Kalogiratou, Th. Monovasilis, and T. E. Simos, "Symplectic integrators for the numerical solution of the Schrödinger equation," Journal of Computational and Applied Mathematics, vol. 158, no. 1, pp. 83-92, 2003.

[17] D. P. Sakas and T. E. Simos, "Multiderivative methods of eighth algebraic order with minimal phase-lag for the numerical solution of the radial Schrödinger equation," Journal of Computational and Applied Mathematics, vol. 175, no. 1, pp. 161-172, 2005.

[18] T. E. Simos, "Exponentially and trigonometrically fitted methods for the solution of the Schrödinger equation," Acta Applicandae Mathematicae, vol. 110, no. 3, pp. 1331-1352, 2010.

[19] T. E. Simos, "Optimizing a hybrid two-step method for the numerical solution of the Schrödinger equation and related problems with respect to phase-lag," Journal of Applied Mathematics, vol. 2012, Article ID 420387, 17 pages, 2012.

[20] Z. A. Anastassi and T. E. Simos, "A parametric symmetric linear four-step method for the efficient integration of the Schrödinger equation and related oscillatory problems," Journal of Computational and Applied Mathematics, vol. 236, no. 16, pp. 3880-3889, 2012.

[21] K. Tselios and T. E. Simos, "Runge-Kutta methods with minimal dispersion and dissipation for problems arising from computational acoustics," Journal of Computational and Applied Mathematics, vol. 175, no. 1, pp. 173-181, 2005.

[22] Z. A. Anastassi and T. E. Simos, "An optimized Runge-Kutta method for the solution of orbital problems," Journal of Computational and Applied Mathematics, vol. 175, no. 1, pp. 1-9, 2005.

[23] D. F. Papadopoulos and T. E. Simos, "A modified RungeKutta-Nyström method by using phase lag properties for the numerical solution of orbital problems," Applied Mathematics \& Information Sciences, vol. 7, no. 2, pp. 433-437, 2013.

[24] T. Monovasilis, Z. Kalogiratou, and T. E. Simos, "Exponentially fitted symplectic Runge-Kutta-Nyström methods," Applied Mathematics \& Information Sciences, vol. 7, no. 1, pp. 81-85, 2013.

[25] Z. Kalogiratou and T. E. Simos, "Newton-Cotes formulae for long-time integration," Journal of Computational and Applied Mathematics, vol. 158, no. 1, pp. 75-82, 2003. 
[26] T. E. Simos, "Closed Newton-Cotes trigonometrically-fitted formulae of high order for long-time integration of orbital problems," Applied Mathematics Letters, vol. 22, no. 10, pp. 16161621, 2009.

[27] T. E. Simos, "New stable closed Newton-Cotes trigonometrically fitted formulae for long-time integration," Abstract and Applied Analysis, vol. 2012, Article ID 182536, 15 pages, 2012.

[28] A. A. Badr, "Finite element method for linear multiterm fractional differential equations," Journal of Applied Mathematics, vol. 2012, Article ID 482890, 9 pages, 2012.

[29] N. J. Ford, J. Xiao, and Y. Yan, "A finite element method for time fractional partial differential equations," Fractional Calculus and Applied Analysis, vol. 14, no. 3, pp. 454-474, 2011.

[30] I. Podlubny, Fractional Differential Equations, vol. 198, Academic Press, San Diego, Calif, USA, 1999.

[31] S. G. Samko, A. A. Kilbas, and O. I. Marichev, Fractional Integrals and Derivatives: Theory and Applications, Gordon and Breach Science, Philadelphia, Pa, USA, 1993.

[32] X. J. Li and C. J. Xu, "A space-time spectral method for the time fractional diffusion equation," SIAM Journal on Numerical Analysis, vol. 47, no. 3, pp. 2108-2131, 2009.

[33] K. Diethelm, "An algorithm for the numerical solution of differential equations of fractional order," Electronic Transactions on Numerical Analysis, vol. 5, no. 1, pp. 1-6, 1997.

[34] K. Diethelm, "Generalized compound quadrature formulae for finite-part integrals," IMA Journal of Numerical Analysis, vol. 17, no. 3, pp. 479-493, 1997.

[35] V. Thomée, Galerkin Finite Element Methods for Parabolic Problems, Springer, Berlin, Germany, 2006. 


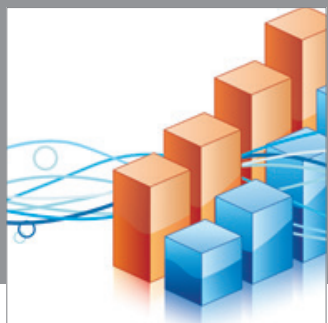

Advances in

Operations Research

mansans

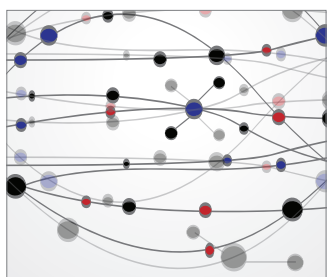

The Scientific World Journal
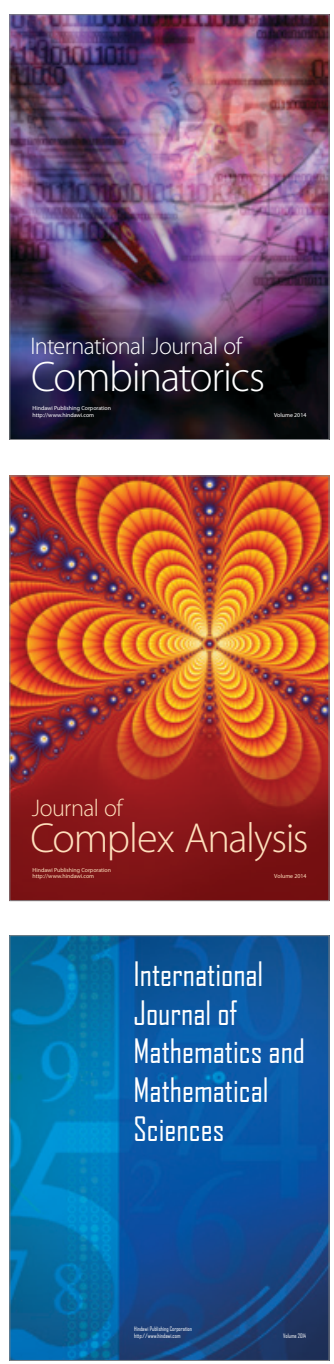
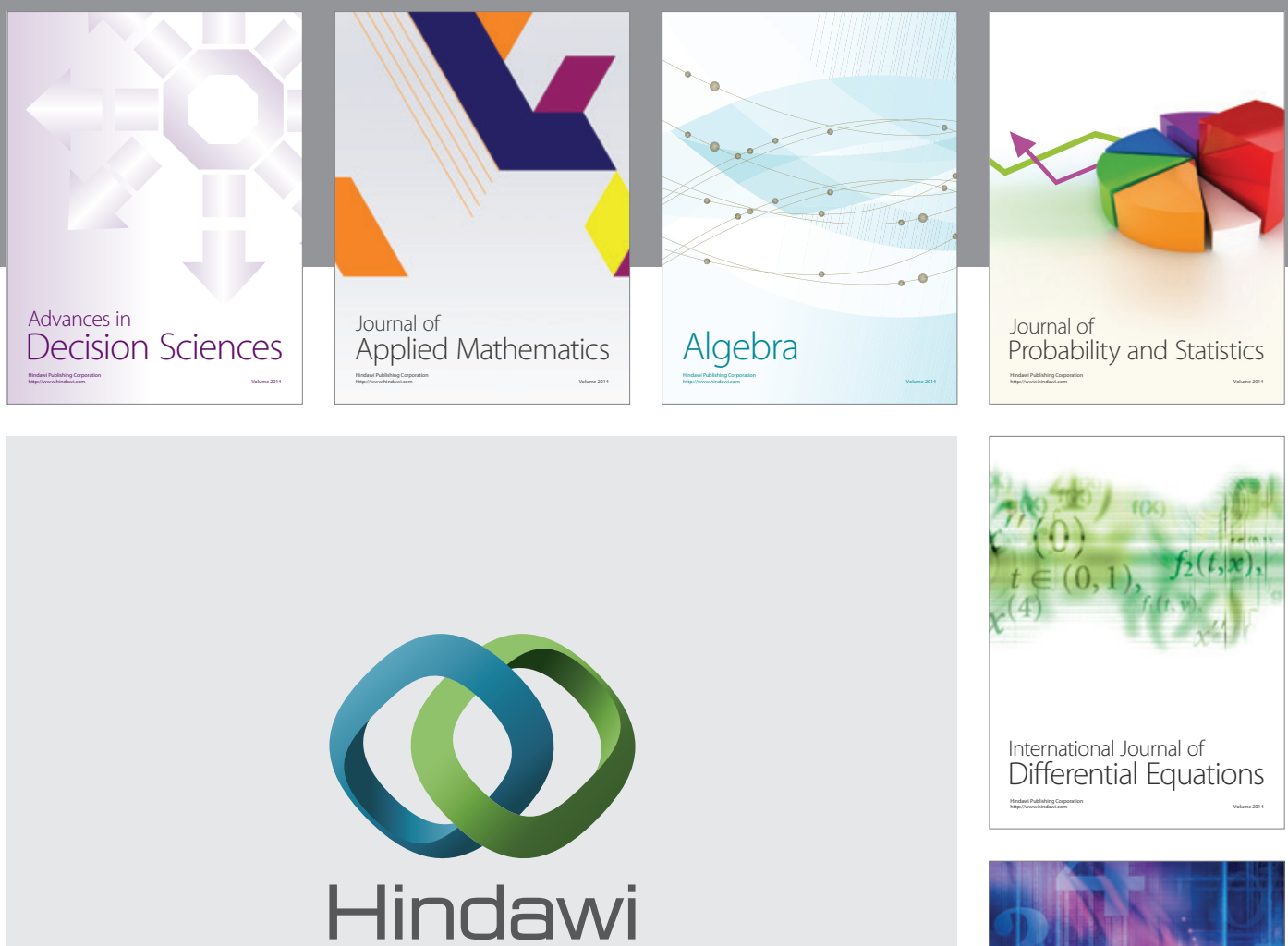

Submit your manuscripts at http://www.hindawi.com
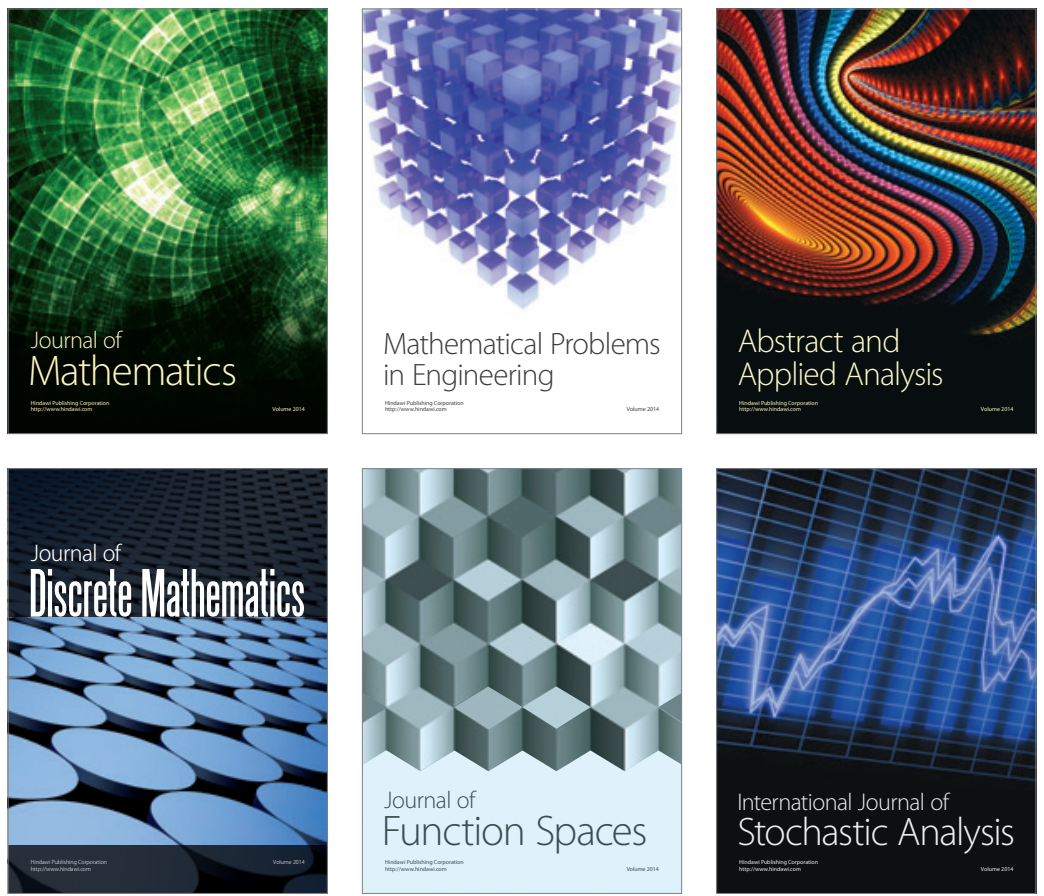

Journal of

Function Spaces

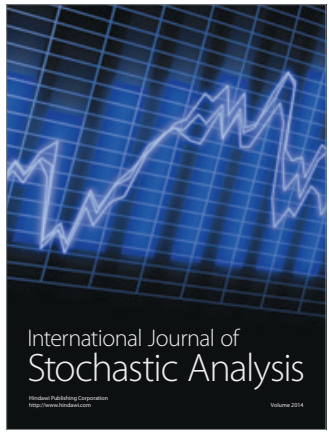

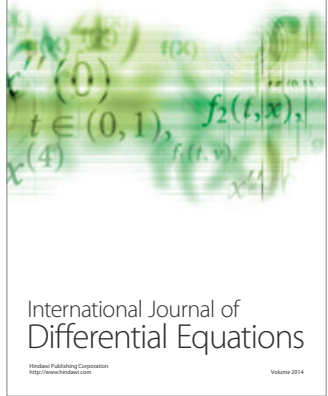
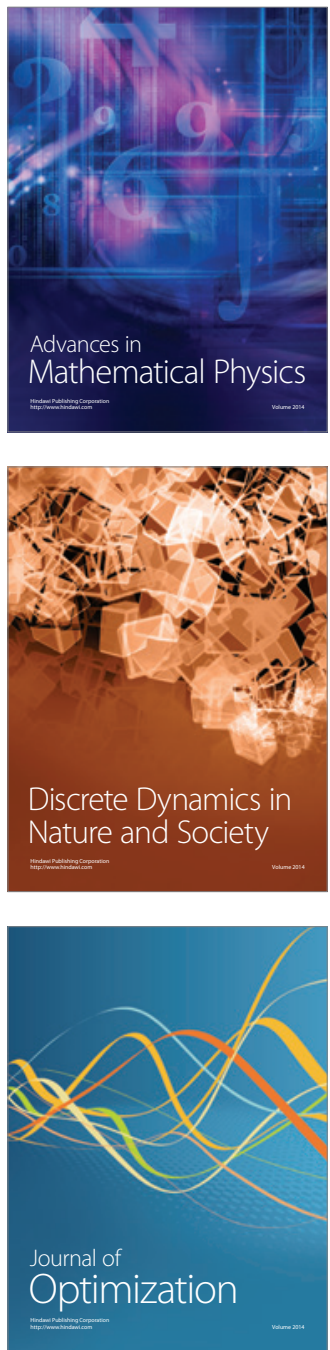